\section{UJMM

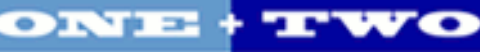

Volume 1| 2009 Spring

\section{Undergraduate Journal of Mathematical}

Modeling: One + Two

2009

\title{
Determination of Azeotropy
}

Kyle Cogswell

University of South Florida

Advisors:

Brian Curtin, Mathematics and Statistics

Scott Campbell, Chemical \& Biomedical Engineering

Problem Suggested By: Scott Campbell

Follow this and additional works at: https://digitalcommons.usf.edu/ujmm

Part of the Mathematics Commons

UJMM is an open access journal, free to authors and readers, and relies on your support:

Donate Now

\section{Recommended Citation}

Cogswell, Kyle (2009) "Determination of Azeotropy," Undergraduate Journal of Mathematical Modeling: One + Two: Vol. 1: Iss. 2, Article 3.

DOI: http://dx.doi.org/10.5038/2326-3652.1.2.3

Available at: https://digitalcommons.usf.edu/ujmm/vol1/iss $2 / 3$ 


\title{
Determination of Azeotropy
}

\begin{abstract}
The ultimate goal of this paper is the determination of an azeotrope within a methanol-acetone system. An azeotrope is the point in a chemical system at which coexisting compositions of vapor and liquid phases are equal. The importance of this point lies in the fact that azeotropes are undesirable; they prevent one from completely separating a mixture through distillation. This state can occur over a range of temperatures and for the purposes of this paper there is only one azeotrope for each given temperature. All azeotropes occur at relative extrema in pressure. By finding these extrema, we find the mole fractions of an azeotrope for a given temperature in the methanol-acetone system.
\end{abstract}

\section{Keywords}

Azeotrope, Methanol acetone system, Kelvin

Creative Commons License

(c) (i) (3)

This work is licensed under a Creative Commons Attribution-Noncommercial-Share Alike 4.0 License. 


\section{Problem Statement}

An azeotrope exists when conditions involving pressure, temperature and mole fractions are just right. Let $X_{1}, X_{2}, P_{1}$ and $P_{2}$ represent, respectively, the mole fractions and partial pressures of methanol and acetone in a given methanol-acetone system. Given the equation, $P=\gamma_{1} X_{1} P_{1}+$ $\gamma_{2} X_{2} P_{2}$, determine the range of temperatures within an overall range of 180 to 500 Kelvin for which an azeotrope will exist for the given methanol-acetone system. A graphical example in which the Azeotrope occurs is depicted below.

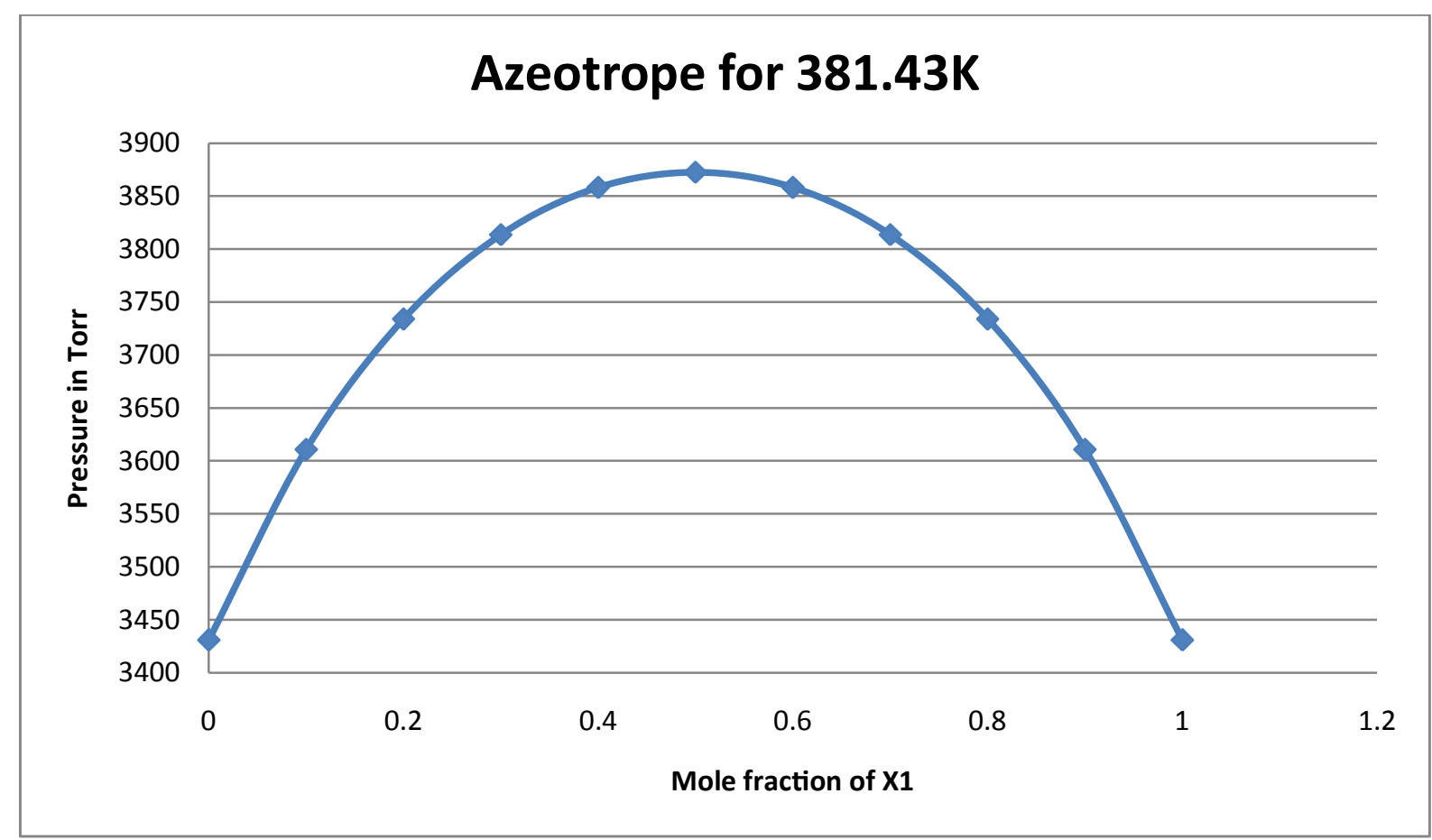

\section{Motivation}

Azeotropy is a common subject in the discipline of chemical engineering. Azeotropes make the very common process of distillation difficult, because one cannot separate the two chemicals involved in the system. Then again, azeotropes only occur at maximum pressures for any given system over a certain temperature range. If one can either raise or lower the temperature enough, the azeotrope will disappear and distillation becomes possible once again. From an engineering standpoint, it is important to be able to identify the azeotropes that can occur in a system so that one can take the proper precautions to avoid them. 


\section{Mathematical Description and Solution Approach}

This problem involves finding the maximum pressure for a methanol-acetone system. Pressure is given by $P=\gamma_{1} X_{1} P_{1}+\gamma_{2} X_{2} P_{2}$ and through the use of differentiation one can then set the derivative to equal 0 and solve for $X$. This may seem complicated at first, but the two $X$ variables are related to one another. $X_{1}$ is equal to $X$ and $X_{2}$ is equivalent to $1-X_{1} . P_{1}$ and $P_{2}$ are related to temperature; one must simply sample a series of temperatures. The equation relating $P_{1}$ (measured in torr) to temperature (measured in Kelvin) is as follows:

$$
P_{1}=\exp \left\{18.5875-\frac{3626.545}{\mathrm{~T}-34.29}\right\}
$$

The relationship of $P_{2}$ to temperature is quite similar:

$$
P_{2}=\exp \left\{16.6513-\frac{2940.46}{\mathrm{~T}-35.93}\right\}
$$

Next come the gamma variables: $\gamma_{1}=e^{A X_{2}^{2}}$ and $\gamma_{2}=e^{A X_{1}^{2}} \cdot A$ is a constant, which varies as the temperature changes according to the function $A=\frac{237.1}{T}-0.1373$. A much shorter version of the differentiation is presented here:

$$
\frac{d P}{d X}=P_{1}\left[X_{1} \frac{d \gamma_{1}}{d X_{1}}+\gamma_{1} \frac{d X_{1}}{d X_{1}}\right]+P_{2}\left[1-X_{1} \frac{d \gamma_{2}}{d X_{1}}+d \gamma_{2} \frac{d\left(1-X_{1}\right)}{d X_{1}}\right]
$$

Setting $d P / d X=0$ gives

$$
0=P_{1}\left[2 A X(1-X)(-1) e^{A(1-X)^{2}}+e^{A(1-X)^{2}}(1)\right]+P_{2}\left[2 A X(1-X) e^{A X^{2}}+e^{A X^{2}}(-1)\right] .
$$

The solution is

$$
X=\frac{1}{2}\left(\frac{\ln \left[\frac{P_{1}}{P_{2}}\right]}{A}+1\right)
$$

A much more detailed version of this derivation is presented in the Appendix.

Using all of the preceding equations, one must determine the range of temperatures within a $180 \mathrm{~K}$ to $500 \mathrm{~K}$ range for which an azeotrope will exist. For the purposes of this experiment, temperatures were sampled every 20 degrees to give a rough estimate of where the azeotropes occurred. After a rough idea was ascertained, additional values of temperature were added to create more data. 


\section{Discussion}

Through the use of the differentiation method to find a maximum value for the pressure function, one can sample a range of temperatures and find the mole fractions that occur at the extreme pressures for each sampled temperature. An azeotrope exists for the temperatures between $290 \mathrm{~K}$ and $465 \mathrm{~K}$. At the first temperature, $290 \mathrm{~K}$, the mole fractions were 0.005611 and 0.994389 for methanol and acetone, respectively. At $465 \mathrm{~K}$, the mole fractions of methanol and acetone were 0.995671 and 0.004329 .

It seems quite reasonable that the mole fractions of the system would change with increasing temperature. The mole fraction of methanol starts out very low, while the mole fraction of acetone starts very high. The mole fraction of methanol decreases with temperature, while the mole fraction of acetone increases with temperature. At exactly $381.43 \mathrm{~K}$, right between the two extremes in temperature, the mole fractions are equal. At the end of the temperature range (465 degrees) the mole fraction of methanol is very high, i.e. close to 1 , while the mole fraction of acetone has decreased to almost 0 .

These results show that over a range of about 290 to $465 \mathrm{~K}$, there exists an azeotrope. An azeotrope is undesirable because it will inhibit distillation. Azeotropy is relevant because the range of temperatures in which azeotropes occur are typical on the earth. On the other hand, if the temperature is dropped below or raised above the temperature range there should be no possibility of an azeotrope existing. In a very cool room or an oven, separating the two chemicals by distillation would, therefore, be simple.

\section{Conclusions}

In conclusion, the azeotrope for the methanol-acetone system can be found by differentiating the original equation $P=\gamma_{1} X_{1} P_{1}+\gamma_{2} X_{2} P_{2}$ and setting the derivative to zero to find the extrema of pressure - in this case, the maximum. The actual values of the temperatures used and the mole fractions that resulted may be found in the appendix.

In an attempt to simplify this paper, an implicit approach was used for the differentiation with substitutions made in the end. This implicit approach — though much simpler in conceptallowed for more errors in the differentiation. In taking this approach, negative signs were lost quite often, leaving one function of pressure equal to the negative value of another. This is not possible, as any pressure function must be positive. Going back to the seemingly more complicated differentiation process was the only answer. Thankfully, multiple parts of the equation cancel out yielding a usable formula for determining the mole fractions for a given temperature.

This problem was not easy, but if one wanted to make it even more challenging, one could ask the same questions using a system with three chemicals. 


\section{Appendix}

The differentiation process is as follows: Let

$$
P=\gamma_{1} X_{1} P_{1}+\gamma_{2} X_{2} P_{2}
$$

With $X_{1}=X$ and $X_{2}=1-X$,

$$
\frac{d P}{d X}=P_{1}\left[X \frac{d \gamma_{1}}{d X}+\gamma_{1} \frac{d X}{d X}\right]+P_{2}\left[(1-X) \frac{d \gamma_{2}}{d X}+d \gamma_{2} \frac{d(1-X)}{d X}\right]
$$

Setting $d P / d X=0$ and differentiating $\gamma_{1}=e^{A X_{2}^{2}}$ and $\gamma_{2}=e^{A X_{1}^{2}}$ gives

$$
\begin{gathered}
0=P_{1}\left[2 A X(1-X)(-1) e^{A(1-X)^{2}}+e^{A(1-X)^{2}}(1)\right]+P_{2}\left[2 A X(1-X) e^{A X^{2}}+e^{A X^{2}}(-1)\right] \Rightarrow \\
\left.0=P_{1}\left[\left(2 A X^{2}-2 A X+1\right)\right) e^{A(1-X)^{2}}\right]+P_{2}\left[\left(-2 A X^{2}+2 A X-1\right) e^{A X^{2}}\right] \Rightarrow \\
P_{1}\left[\left(2 A X^{2}-2 A X+1\right) e^{A(1-X)^{2}}\right]=-P_{2}\left[\left(-2 A X^{2}+2 A X-1\right) e^{A X^{2}}\right] \Rightarrow \\
P_{1}\left[\left(2 A X^{2}-2 A X+1\right) e^{A(1-X)^{2}}\right]=-P_{2}(-1)\left[\left(2 A X^{2}-2 A X+1\right) e^{A X^{2}}\right] \Rightarrow \\
P_{1}\left[\left(2 A X^{2}-2 A X+1\right) e^{A(1-X)^{2}}\right]=P_{2}\left[\left(2 A X^{2}-2 A X+1\right) e^{A X^{2}}\right] \Rightarrow \\
P_{1} e^{A(1-X)^{2}}\left[\left(2 A X^{2}-2 A X+1\right)\right]=P_{2} e^{A X^{2}}\left[\left(2 A X^{2}-2 A X+1\right)\right] \Rightarrow \\
\frac{P_{1}}{P_{2}}=e^{A X^{2}-A(1-X)^{2}} \Rightarrow \ln \left[\frac{P_{1}}{P_{2}}\right]=A X^{2}-A(1-X)^{2}=A X^{2}-\left[1 A-2 A X+A X^{2}\right] \\
\Rightarrow \ln \left[\frac{P_{1}}{P_{2}}\right]=A(2 X-1) .
\end{gathered}
$$

Therefore,

$$
X=\frac{1}{2}\left(\frac{1}{A} \ln \left[\frac{P_{1}}{P_{2}}\right]+1\right)
$$


Temperature and Mole Fractions of Occurring Azeotropes

\begin{tabular}{|c|c|c|c|c|c|c|}
\hline & 1 & 2 & A & & $\mathrm{X} 1$ & \\
\hline 180 & 0.001834 & 0.023315 & 1.179922 & -2.54268 & -0.57748 & 1.57747 \\
\hline 200 & 036978 & 280639 & 1.0482 & -2.02673 & -0.46677 & \\
\hline 220 & 0.390422 & 1.96721 & 0.940427 & -1.61714 & -0.35979 & .35979 \\
\hline 240 & 2.606661 & 9.414252 & 0.850617 & -1.28415 & -0.25484 & .25483 \\
\hline 260 & 2.4311 & 4.06762 & 774623 & 15 & 073 & 15073 \\
\hline 280 & 45.97177 & 99.85216 & 0.709486 & -0.77566 & -0.04664 & 1.0466 \\
\hline 290 & 742 & 160.4328 & 286 & -0.6 & 611 & \\
\hline 300 & 139.6268 & 248.6749 & 0.653033 & -0.57 & 0.058083 & 191 \\
\hline 310 & 649 & 97 & 0.627 & 43 & 10837 & 005 \\
\hline 320 & 362.9934 & 544.6367 & 0.603638 & -0.40573 & 0.163925 & .83607 \\
\hline 330 & 557.5915 & 774.4214 & 0.581185 & -0.3 & 0.217397 & $0.7826 C$ \\
\hline 340 & 832.7945 & 1075.952 & 0.560053 & -0.2 & 0.271295 & 0.72870 \\
\hline 350 & 1212.615 & 1463.908 & 0.540129 & $-0.1 \varepsilon$ & 0.325661 & 0.67433 \\
\hline 360 & 1725.391 & 954.259 & 0.521311 & -0.1 & 0.380534 & .61946 \\
\hline$\underline{370}$ & 2403.96 & 2564.124 & 0.503511 & 645 & 0.43595 & $0.564 C$ \\
\hline 380 & 3285.747 & 3311.611 & 0.486647 & -0.0 & 0.491944 & 0.5080 \\
\hline 81.43 & 3430.846 & 3430.845 & 484308 & 2.2 & 0.5 & \\
\hline 85 & 3815.798 & 42.85 & 478544 & 0.01 & 0.520168 & 983 \\
\hline$\underline{390}$ & 4412.765 & & 0.470649 & 0.04 & 549 & 0.4 \\
\hline 400 & 5831.538 & & & & 0.605796 & 0.39420 \\
\hline 410 & 592.95 & 6572.035 & 0.440993 & 0.1 & 3718 & 0.33628 \\
\hline 420 & 9752.012 & 8064.667 & 0.427224 & 0.189981 & 0.722344 & 0.27765 \\
\hline 430 & 12367.58 & 9794.039 & 0.414095 & 0.23 & 0.781704 & 0.21829 \\
\hline 440 & 15502.03 & 11780.43 & 0.401564 & 0.274531 & 0.841828 & 0.15817 \\
\hline 450 & 19220.84 & 14043.86 & 0.389589 & 0.31381 & 0.902745 & 0.09725 \\
\hline 460 & 23592.23 & 16603.98 & 0.378135 & 0.351275 & 0.964484 & 0.0355 \\
\hline 465 & 26044.59 & 18001.3 & 0.372592 & 0.369366 & 0.995671 & 0.00432 \\
\hline 470 & 28686.69 & 19479.91 & 0.367168 & 0.387049 & 1.027074 & -0.0270 \\
\hline 480 & 34576.56 & 22690.15 & 0.356658 & 0.421245 & 1.090544 & -0.0905 \\
\hline 490 & 41335.56 & 26252.44 & 0.346578 & 0.453964 & 1.154924 & -0.154 \\
\hline 500 & 49038.34 & 30183.71 & 0.3369 & 0.4853 & 1.220244 & -0.220 \\
\hline
\end{tabular}


Data for Locus Graph:

\begin{tabular}{|c|c|c|c|c|c|}
\hline \multicolumn{3}{|c|}{$\mathbf{T}=\mathbf{3 7 0} \mathrm{K}$} & \multicolumn{3}{|c|}{$\mathrm{T}=381.43 \mathrm{~K}$} \\
\hline$X_{1}$ & $X_{2}$ & Pressure & $X_{1}$ & $X_{2}$ & Pressure \\
\hline 0 & 1 & 2564.124209 & 0 & 1 & 3430.845253 \\
\hline 0.1 & 0.9 & 2680.813443 & 0.1 & 0.9 & 3610.642186 \\
\hline 0.2 & 0.8 & 2756.633719 & 0.2 & 0.8 & 3733.865635 \\
\hline 0.3 & 0.7 & 2801.086769 & 0.3 & 0.7 & 3813.510872 \\
\hline 0.43595 & 0.56405 & 2821.618089 & 0.4 & 0.6 & 3858.094666 \\
\hline 0.5 & 0.5 & 2817.260203 & 0.5 & 0.5 & 3872.43618 \\
\hline 0.6 & 0.4 & 2792.863896 & 0.6 & 0.4 & 3858.094799 \\
\hline 0.7 & 0.3 & 2745.268874 & 0.7 & 0.3 & 3813.511144 \\
\hline 0.8 & 0.2 & 2670.108213 & 0.8 & 0.2 & 3733.866056 \\
\hline 0.9 & 0.1 & 2560.020008 & 0.9 & 0.1 & 3610.642772 \\
\hline 1 & 0 & 2403.960182 & 1 & 0 & 3430.846028 \\
\hline & $T=390 \mathrm{~K}$ & & & & \\
\hline$X_{1}$ & $X_{2}$ & Pressure & & & \\
\hline 0 & 1 & 4215.645 & & & \\
\hline 0.1 & 0.9 & 4458.043 & & & \\
\hline 0.2 & 0.8 & 4629.379 & & & \\
\hline 0.3 & 0.7 & 4745.836 & & & \\
\hline 0.4 & 0.6 & 4818.219 & & & \\
\hline 0.548549 & 0.451451 & 4857.013 & & & \\
\hline 0.6 & 0.4 & 4852.335 & & & \\
\hline 0.7 & 0.3 & 4815.315 & & & \\
\hline 0.8 & 0.2 & 4736.79 & & & \\
\hline 0.9 & 0.1 & 4607.427 & & & \\
\hline 1 & 0 & 4422.801 & & & \\
\hline
\end{tabular}




\section{Azeotropic Data for 370K, 381.43K, and 390K:}

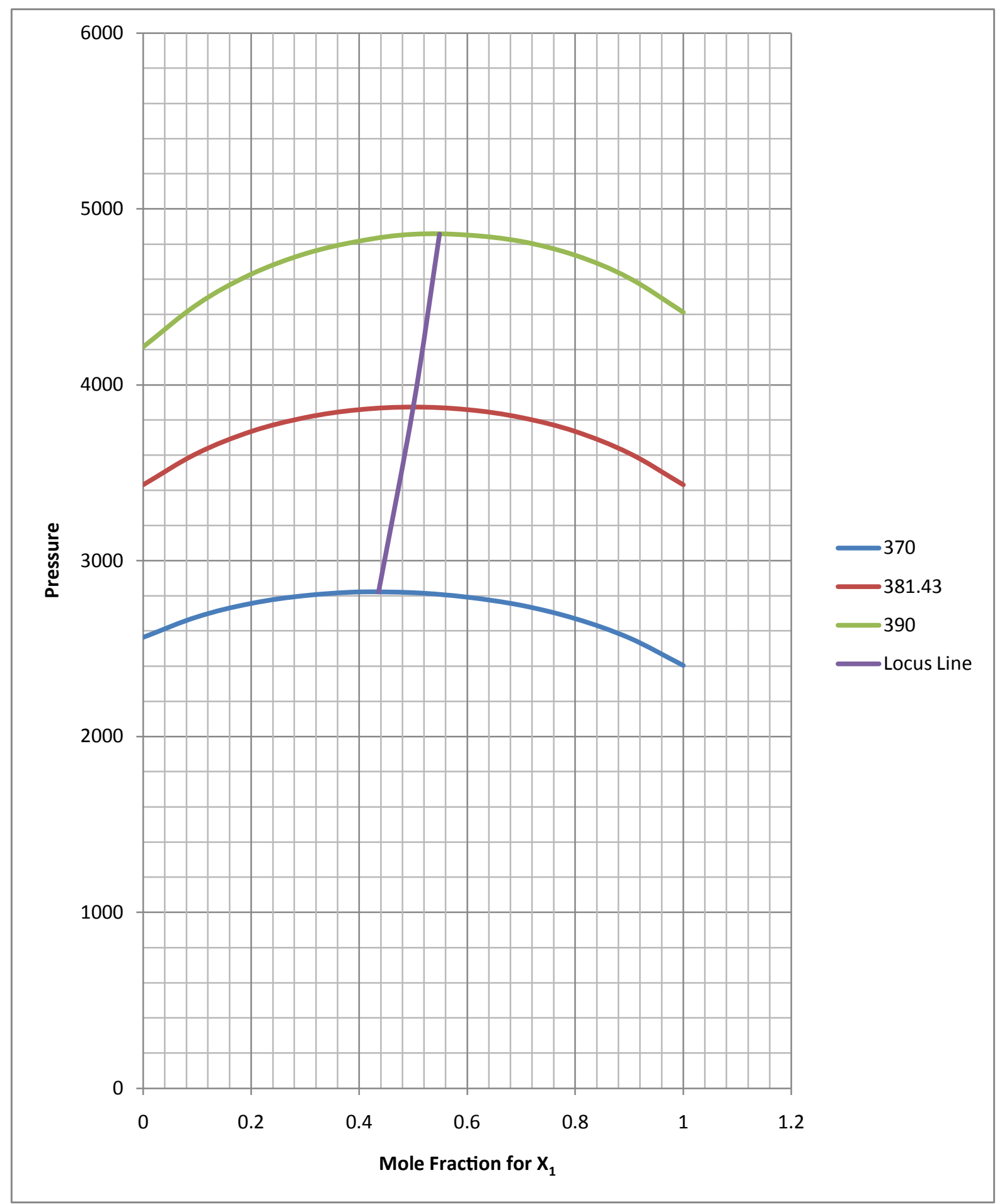

